Plasma Science and Applications (ICPSA 2013)

International Journal of Modern Physics: Conference Series

Vol. 32 (2014) 1460319 (10 pages)

(C) The Author

DOI: $10.1142 / \mathrm{S} 2010194514603196$

\title{
RF plasma polymerised thin films from natural resources
}

\author{
Mohan V. Jacob* \\ Electronic Materials Refind Lab, School of Engineering and Physical Sciences, \\ James Cook University, Townsville, 4811, Australia \\ "Mohan.Jacob@jcu.edu.au \\ Chris D. Easton \\ CSIRO, Sydney, Australia \\ chris.easton@csiro.com \\ Liam J. Anderson \\ Electronic Materials Refind Lab, School of Engineering and Physical Sciences, \\ James Cook University, Townsville, 4811, Australia \\ Kateryna Bazaka \\ Electronic Materials Refind Lab, School of Engineering and Physical Sciences, \\ James Cook University, Townsville, 4811, Australia \\ Published 13 August 2014
}

\begin{abstract}
Plasma polymerisation is an effective tool for fabrication of thin films from volatile organic monomers. RF plasma assisted deposition is used for one-step, chemical-free polymerisation of nonsynthetic materials derived directly from agricultural produces. By varying the deposition parameters, especially the input RF power, the film properties can be tailored for a range of uses, including electronics or biomedical applications. The fabricated thin films are optically transparent with refractive index close to that of glass. Given the diversity of essential oils, this paper compares the chemical and physical properties of thin films fabricated from several commercially exploited essential oils and their components. It is interesting to note that some of the properties can be tailored for various applications even though the chemical structure of the derived polymer is very similar. The obtained material properties also show that the synthesised materials are suitable as encapsulating layers for biodegradable implantable metals.
\end{abstract}

Keywords: Plasma polymer; thin Film; transparent thin films.

\section{Introduction}

Radio Frequency (RF) plasma polymerization is a low-cost, versatile technique for fabricating organic thin films from a wide array of organic and synthetic materials, including those that do not normally polymerize via conventional pathways. ${ }^{1-4}$ of particular interest is the utility of this method in one-step deposition of high-quality

This is an Open Access article published by World Scientific Publishing Company. It is distributed under the terms of the Creative Commons Attribution 3.0 (CC-BY) License. Further distribution of this work is permitted, provided the original work is properly cited. 


\section{V. Jacob et al.}

coatings from natural resources, e.g. plant essential oils, without the need for additional chemical processing. ${ }^{1-3}$ Plasma polymerized thin films have found a host of applications, from electronics to biomedical devices. ${ }^{4}$ In biomedical field, plasma coatings can be used to afford material chemical or mechanical protection, impart biocompatibility and antimicrobial activity, and for drug delivery. ${ }^{5,}{ }^{6}$ In electronics, films from organic precursors have been used to replace conventional inorganic materials to produce superior organic and hybrid devices.

Organic electronics offer several advantages over conventional silicon electronics, such as flexibility and low cost fabrication from solution, allowing production of flexible, lightweight, self-illuminating displays based on Organic Light Emitting Diode (OLED) and Organic Field Effect Transistor (OFET) devices and low-cost Organic Photo-Voltaic (OPV) and sensors. ${ }^{7-10}$ Organic displays exhibit high brightness, fast response times, wide viewing angles and low operating voltages. Over the last few years, a number of organic electronics devices have become commercially available and many outperform their conventional counterparts. ${ }^{10-13}$

We have fabricated plasma polymer thin films from essential oils such as Lavender Oil ${ }^{14-17}$ and Tea Tree oil and several of their components. ${ }^{2,18-20}$ The aim of this paper is to compare the chemical, optical, surface and physical properties of the polymer thin films fabricated from the major components found in these essential oils.

\section{Experimental}

A custom-made glass chamber was used to fabricate the thin films from various essential oil components. Capacitively coupled Copper electrodes are used to ignite glow discharge from $13.56 \mathrm{MHz}$ RF generator with an output power of $25 \mathrm{~W}$. The distance between the electrodes was adjusted to $10 \mathrm{~cm}$ in order to achieve optimal deposition conditions. Super-white glass substrates $(25 \mathrm{~mm} \times 75 \mathrm{~mm})$ were washed in Extran and distilled water. The substrates were subjected to 30 minute of ultrasonic cleaning in distilled water heated to $50^{\circ} \mathrm{C}$ followed by rinsing in Propan-2-ol and air-dry. The cleaned glass substrate was placed into the deposition chamber and the chamber was flushed with argon for 1 minute. The chamber was then evacuated to achieve a pressure of 100 mTorr. The monomer was released gradually into the chamber, the glow was maintained by controlling the monomer flow by means of a vacuum stop cock. Deposition was performed at room temperature, pressure of 100 mTorr.
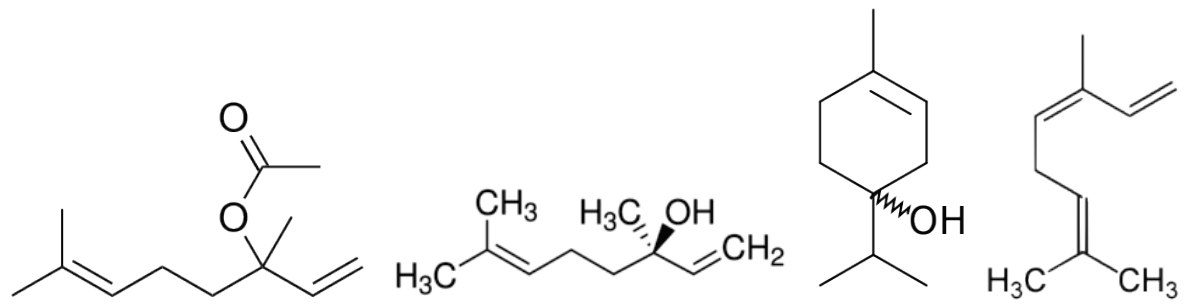

Fig. 1. Chemical structure of linalyl acetate, linalool acetate, terpene-4-ol and cis- $\beta$-ocimene. 
We have used monomers Lavender oil, linalyl acetate $\left(\mathrm{CH}_{3} \mathrm{CO}_{2} \mathrm{C}\left(\mathrm{CH}=\mathrm{CH}_{2}\right)\left(\mathrm{CH}_{3}\right) \mathrm{CH}_{2} \mathrm{CH}_{2} \mathrm{CH}=\mathrm{C}\left(\mathrm{CH}_{3}\right)_{2}\right.$, Molecular Weight 196.29), linalool acetate $\left(\left(\mathrm{CH}_{3}\right)_{2} \mathrm{C}=\mathrm{CHCH}_{2} \mathrm{CH}_{2} \mathrm{C}\left(\mathrm{CH}_{3}\right)(\mathrm{OH}) \mathrm{CH}=\mathrm{CH}_{2}\right.$, Molecular Weight 154.25), terpene4-ol $\left(\mathrm{C}_{10} \mathrm{H}_{18} \mathrm{O}\right.$, Molecular Weight 154.25) and cis- $\beta$-ocimene $\left(\mathrm{C}_{10} \mathrm{H}_{16}\right.$, Molecular Weight 136.24) for this study. Fig. 1 shows the chemical structure of linalyl acetate, linalool acetate, terpene-4-ol and cis- $\beta$ ocimene.

Spectroscopic ellipsometry will be used to determine the thickness and optical constants of the thin films, including refractive index, extinction coefficient and transmission. The chemical structure of the polymers will be examined using Fourier transform infra-red (FTIR) spectroscopy. The surface of the polymers will be investigated by atomic force microscope (AFM), and water contact angle (WCA) measurements.

\section{Results and Discussion}

Spectroscopic ellipsometry was used to determine the thickness and optical properties of the polymer thin films including refractive index, extinction coefficient and transmission. Measurements were performed in the wavelength range $190-1000 \mathrm{~nm}$ using a variable angle spectroscopic ellipsometer (model M-2000, J. A. Woollam Co., Inc.). Ellipsometric parameters $\Psi$ and $\Delta$ were obtained at three different angles of incidence, $\varphi=55^{\circ}, 60^{\circ}$, and $65^{\circ}$, in addition to transmission data. These measured parameters were used to derive the optical constants based on a model of the sample built in the software WVASE32. A Gaussian and/or Tauc-Lorentz oscillator were employed to obtain the best fit to the data, with a lower mean-squared error.

\subsection{Deposition rate}

Polymer thin films were successfully fabricated from Lavender oil and several common essential oil components under the similar deposition parameters. Thickness of the resultant polymers (shown in Fig. 2) was determined using spectroscopic ellipsometry. The rate of deposition varied from monomer to monomer. Linalyl acetate exhibited lowest rate of deposition $(25 \mathrm{~nm} / \mathrm{min})$ and cis- $\beta$-ocimene exhibited highest rate of deposition $(100 \mathrm{~nm} / \mathrm{min})$. Based on the graph we can also infer that more than one component polymerized when Lavender oil was used, the oil typically consisting of over 60 components.

\subsection{Optical properties}

The refractive index and extinction coefficient of the thin films were examined by employing spectroscopic ellipsometry (Fig. 3). The refractive indices of the thin films were approximately equal and were slightly greater than that of glass, with the exception of the cis- $\beta$-ocimene based polymer. The refractive index of the cis- $\beta$-ocimene based polymer however was greater than the other polymers, especially at higher wavelengths. The transmission measurements confirmed that all the polymers were optically transparent (Fig. 4). The extinction coefficient values also confirmed the optical 
transparency of the fabricated samples. Our studies also verified that the thin films do not have thickness dependence of the refractive index.

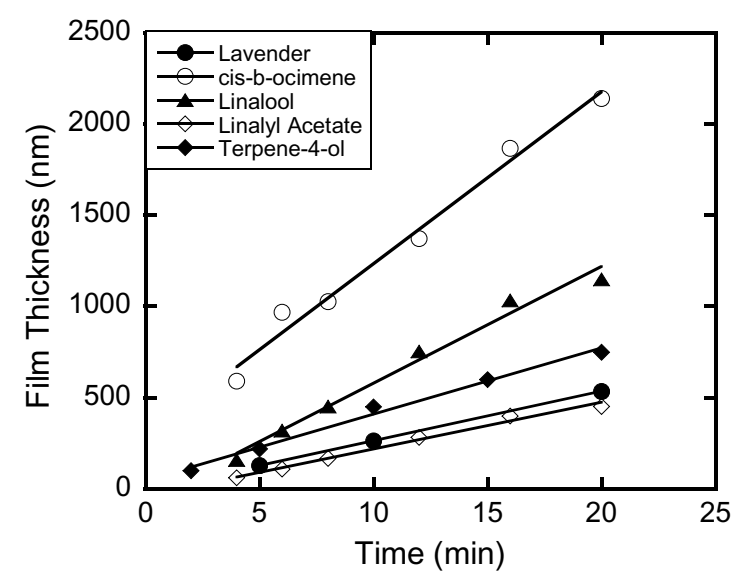

Fig. 2. Film thickness of resultant polymers as a function of time.

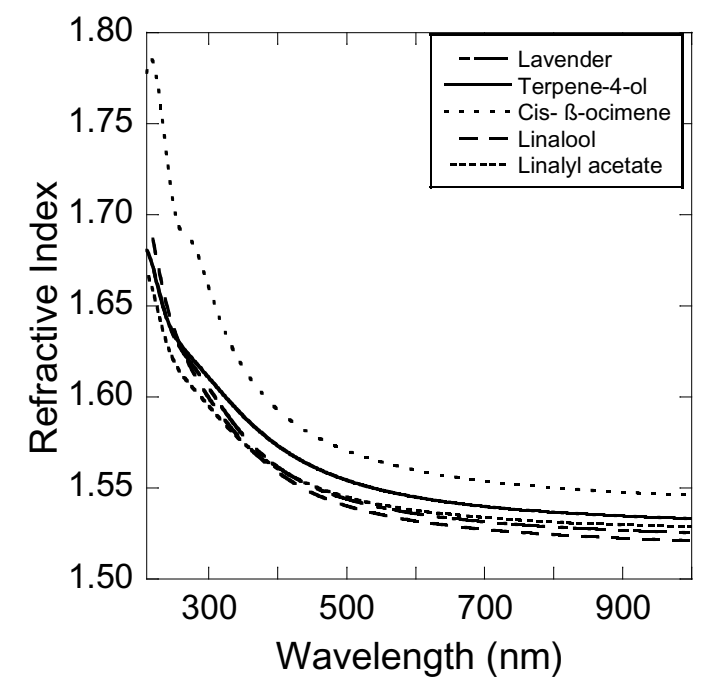

Fig. 3. The refractive index profiles of the polymer thin films.

\subsection{Chemical structural analysis}

A Perkin-Elmer Spectrum 2000 FTIR Spectrometer (RMIT) was employed to determine the chemical structure of the polymer thin films. Spectra were obtained in transmission mode in the region of $4000-500 \mathrm{~cm}^{-1}$. A total of 32 scans were acquired for each film at a resolution of $1 \mathrm{~cm}^{-1}$. $\mathrm{CO}_{2}$ and $\mathrm{H}_{2} \mathrm{O}$ were accounted for in the spectra by a background subtraction procedure using a pre-recorded background under the same atmospheric conditions. 


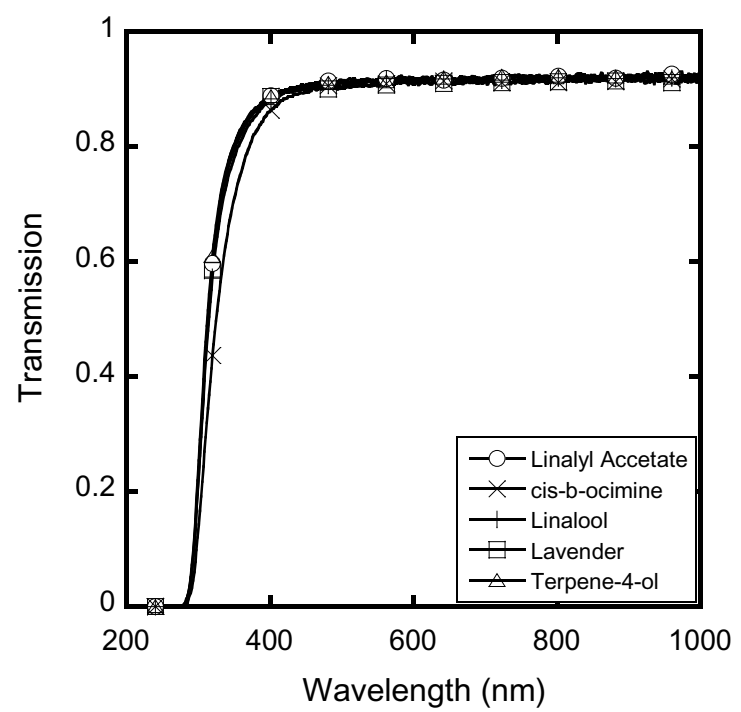

Fig. 4. Optical properties of the polymer thin films.

FTIR spectra of the monomer (top panel) and resultant polymer (bottom panel) for the four components studied are presented in Fig. 5. It is apparent that irrespective of the starting monomer, the spectra of the resultant polymers are similar. The majority of the same spectral peaks are found in each polymer, with some variation in the relative intensity of the peaks. All polymers demonstrate peaks associated with the presence of alcohol ( 3460 $\left.\mathrm{cm}^{-1}, \mathrm{O}-\mathrm{H}\right)$, and methyl $\left(2956 \mathrm{~cm}^{-1}, 2871 \mathrm{~cm}^{-1}, 1457 \mathrm{~cm}^{-1}, 1376 \mathrm{~cm}^{-1}\right)$ and methylene $\left(2930 \mathrm{~cm}^{-1}, 1457 \mathrm{~cm}^{-1}\right)$ groups. A large number of strong methyl peaks suggests that the thin films are comprised of a large number of short polymer chains, which is characteristic of plasma polymers. A $\mathrm{C}=\mathrm{O}$ peak associated with a ketone can be identified in the spectra of all the polymers with the exception of the cis- $\beta$-ocimene based polymer.

We can infer from this information that for the ketone peak to be present in the resulting polymer, the monomer must contain an oxygen functional group. However, as the $-\mathrm{OH}$ peak is present for all polymers then it is likely that incorporation of $-\mathrm{OH}$ into the polymer structure is resulting from multiple sources, most likely due to oxygen uptake and the subsequent radical termination post deposition regardless of the chemical composition of the monomer. As expected there is a correlation between oxygen content of monomer and content in resultant polymers. With the exception of the very weak -OH peak which is likely the result of radical termination, the structure consists of peaks allocated to $\mathrm{C}=\mathrm{C}$ stretching $\left(1604 \mathrm{~cm}^{-1}\right)$ and $\mathrm{C}-\mathrm{H}$ stretching connected to $\mathrm{C}=\mathrm{C}$ bonding $\left(3020 \mathrm{~cm}^{-1}\right)$. Therefore, in addition to the lack of ketone peak, the chemical structure of the cis- $\beta$-ocimene based polymer is the only spectra to exhibit any significant deviation compared to the other samples. The FTIR spectra of the polymers obtained in this study can be compared with the Lavender oil based polymer; with the exception of the cis- $\beta$ - 
ocimene based polymer, the spectra of all the polymers are similar. The only significant difference occurs in the intensity of the $-\mathrm{OH}$ and $\mathrm{C}=\mathrm{O}$ peaks.
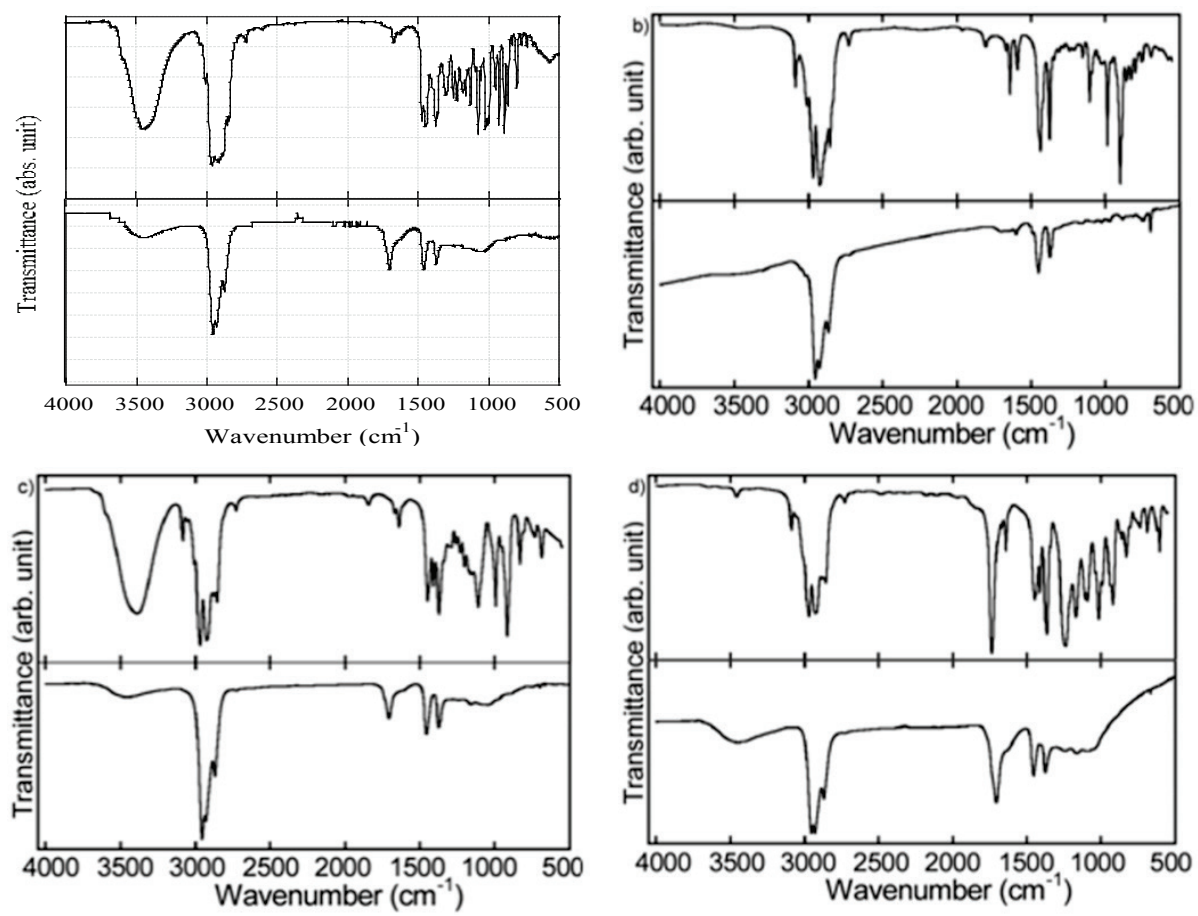

Fig. 5. FTIR spectra for the components in monomer form (top panel) and resultant polymer thin films (bottom panel) fabricated from a) terpene-4-ol, b) cis- $\beta$-ocimene, c) linalool, d) linalyl acetate.

\subsection{Surface topology analysis}

Analysis of the surface morphology was undertaken at the AAC, JCU using the NT-MDT NTEGRA Prima AFM in semi-contact mode.

AFM images of the resultant polymers were obtained to understand the surface topology. Images of the films of scan size $1 \times 1 \mu \mathrm{m}$ are presented in Fig. 6 a)- e). From these images it is apparent that the surface topologies of all the films with exception of cis- $\beta$-ocimene based polymer were similar. The linalyl acetate based polymers produced the smoothest surfaces, with a roughness comparable with that of the Lavender oil based polymer fabricated under the same conditions $(0.42 \mathrm{~nm})$. The cis- $\beta$-ocimene based polymer resulted in a surface roughness much higher than the other polymers $(14.82 \mathrm{~nm})$. The higher rate of deposition of cis- $\beta$-ocimene can account for the higher surface roughness. 

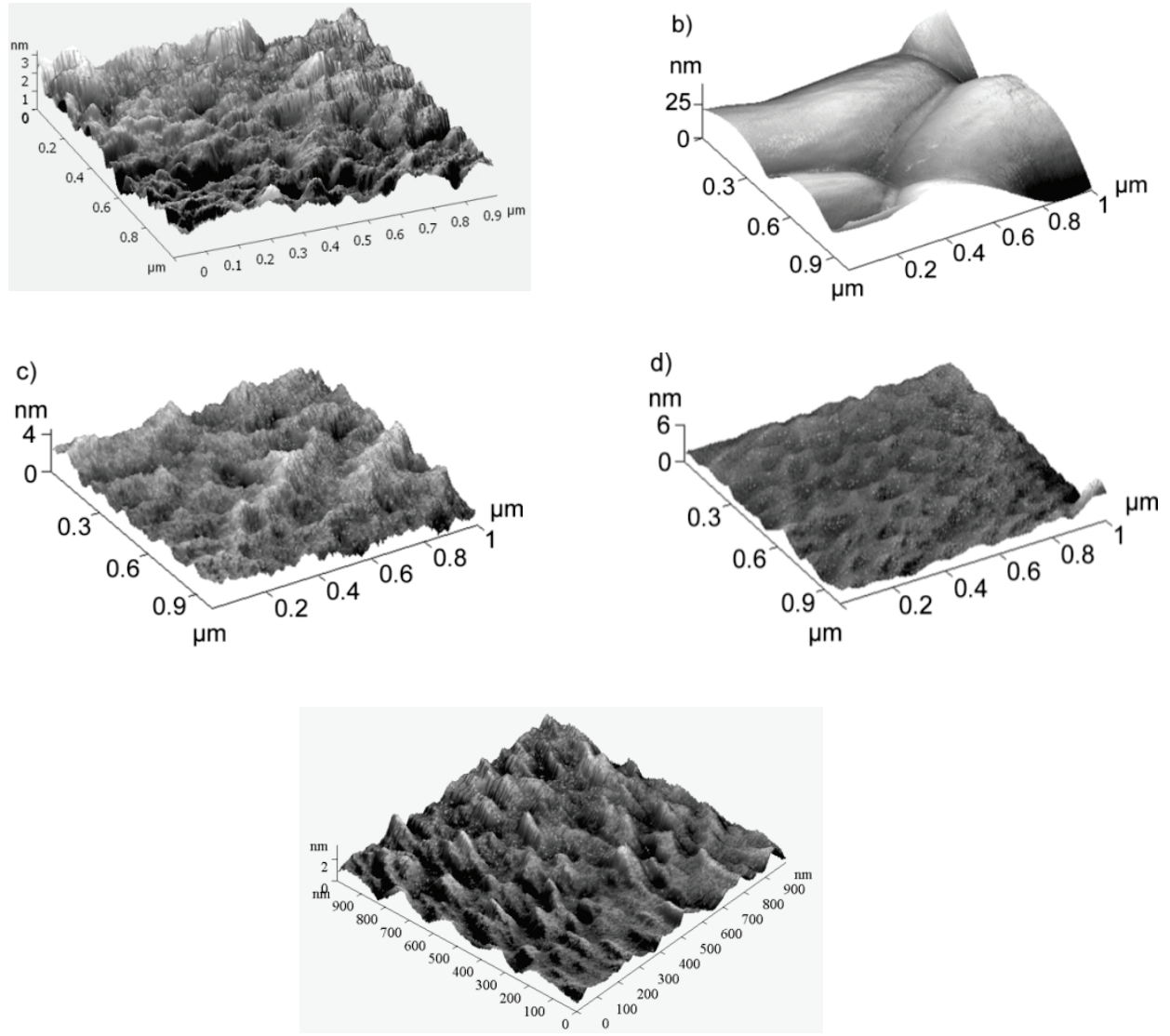

Fig. 6. AFM images of the polymer films in semi-contact mode: a) Lavender oil, b) cis- $\beta$-ocimene, c) linalool, d) linalyl acetate, e) terpene-4-ol.

\subsection{Water contact angle}

A KSV 101 system was implemented for the water contact angle measurements. Drop volume of approximately $8 \mu \mathrm{L}$ was employed, where 8 drops were used to determine the water contact angle (WCA) for each film. Once the drop was placed on the surface, the KSV CAM software was triggered and an image was recorded every second for $30 \mathrm{sec}$ in order to monitor the contact angle as a function of time. Using the image processing software, contact angle values were obtained by fitting the measured drop profile with the Young-Laplace equation.

Equilibrium water contact angle values were obtained for the fabricated polymers (Fig. 7). The WCA ranges from $68.5^{\circ}$ (terpene-4-ol) to $94.14^{\circ}$ (cis- $\beta$-ocimene). Referring back to the FTIR analysis, as expected the polymers with strong peaks assigned to oxygen resulted in low WCA values, while the cis- $\beta$-ocimene based polymer with very 
little oxygen measured the highest WCA value. The contact angle observed for the cis- $\beta$ ocimene based polymer could also be attributed to the relatively large average roughness of the film. An increase in surface roughness has been previously associated with an increase in hydrophobicity ${ }^{21}$. The polymer with a WCA closest to the Lavender oil based polymer $\left(83.64^{\circ}\right)$ is the linalyl acetate polymer $\left(85.72^{\circ}\right)$.

An initial high rate of change in contact angle typically represents absorption of water by the sample. From Fig. 7, there is no apparent rapid change in contact angle for most of the polymers. From the rate of variation of WCA data, we can assume that the amount of water absorption into the polymer was expected to be minimal.

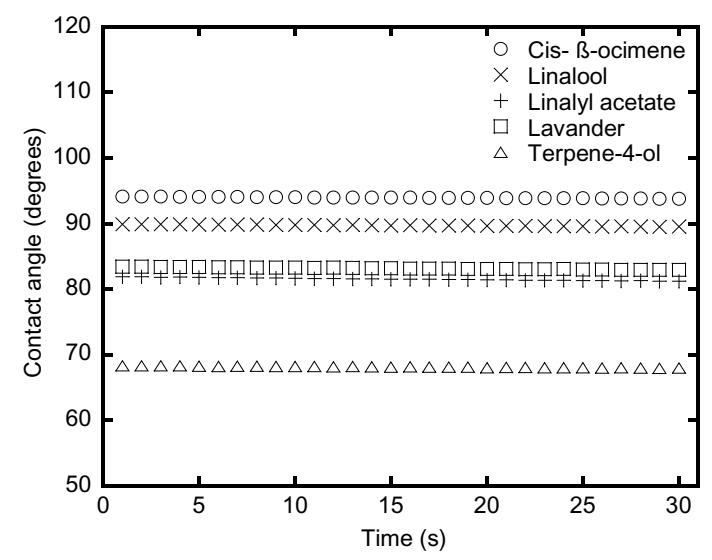

Fig. 7. Water contact angle of the resultant films as a function of time.

WCA as a function of time can provide information concerning reactions between the liquid and the polymer, where a large rate of change is associated with reorientation of functionalities at the solvent-surface interface. The WCA results demonstrate that it is highly unlikely that interactions such as swelling or reorientation of polar groups are occurring at the liquid-solid interface, and as such these polymers are stable while in contact with water. In addition, the WCA for the polymers, with the exception of cis- $\beta$ ocimene, fall within the demonstrated range for plasma polymers that provide favorable dose response of I-protein A uptake to human immunoglobulin G (hIgG) ${ }^{22}$. It has also been shown that linalool can induce a decrease in biofilm metabolic activity ${ }^{23}$. Therefore these materials have the potential to be implemented in biomedical applications as either bio-reactive or bio-non-reactive coatings.

\section{Conclusion}

This paper compares the Plasma polymer thin films from Lavender oil and 4 major constituents of essential oils. The adopted fabrication technique allows fast deposition of polymer thin films, at approximately 25 to $100 \mathrm{~nm} / \mathrm{min}$. FTIR spectra of the polymers 
have been obtained for both the monomers and resulting polymers. It is apparent that irrespective of the starting monomer, the spectra of the resultant polymers are very similar. The cis- $\beta$-ocimene based polymer exhibited the most significant deviation as the spectral peak for ketone at approximately $1710 \mathrm{~cm}^{-1}$ was absent, and peaks associated with $\mathrm{C}=\mathrm{C}$ were present. The results indicate that an oxygen functional group contained in the monomer was required in order to obtain a ketone peak in the resulting polymer; however this requirement was not necessary for the -OH stretch. All polymers were proven to be optically transparent. Refractive index of the polymers was only slightly greater than that of glass, with the exception of the cis- $\beta$-ocimene based polymer which was greater still. AFM investigation of the polymers demonstrated some variation in the surface topology between the thin films. The average roughness of most of the polymers was around $0.5 \mathrm{~nm}$. The cis- $\beta$-ocimene based polymer however has a surface topology that results in a much higher average roughness $(14.82 \mathrm{~nm})$. WCA values ranged from $68.5^{\circ}$ to $94.14^{\circ}$ indicating that the polymers ranged from hydrophilic to mildly hydrophobic. The WCA results demonstrated that the polymer thin films were stable while in contact with water.

\section{Acknowledgments}

KB acknowledges the ARC DECRA (DE130101550) fellowship.

\section{References}

1. K. Bazaka, M. V. Jacob, V. K. Truong, F. Wang, W. A. A. Pushpamali, J. Y. Wang, A. V. Ellis, C. C. Berndt, R. J. Crawford and E. P. Ivanova, Biomacromolecules 11 (8), 2016 (2010).

2. M. V. Jacob, K. Bazaka, D. Taguchi, T. Manaka and M. Iwamoto, Chem. Phys. Lett. 528, 26 (2012).

3. M. V. Jacob, N. S. Olsen, L. Anderson, K. Bazaka and R. A. Shanks, Thin Solid Films 546, 167 (2013).

4. K. Ostrikov, E. Neyts and M. Meyyappan, Advances in Physics 62 (2), 113 (2013).

5. K. Bazaka, M. Jacob, R. Crawford and E. Ivanova, Appl Microbiol Biotechnol 95 (2), 299 (2012).

6. K. Bazaka, M. V. Jacob, R. J. Crawford and E. P. Ivanova, Acta Biomater. 7 (5), 2015 (2011).

7. Y. Li, B. W. Muir, C. D. Easton, L. Thomsen, D. R. Nisbet and J. S. Forsythe, Applied Surface Science 288, 288 (2014).

8. I. McCulloch, Advanced Materials 25 (13), 1811 (2013).

9. P. Schwamb, T. C. Reusch and C. J. Brabec, Organic Electronics (2013).

10. F. Zhang, C. a. Di, N. Berdunov, Y. Hu, Y. Hu, X. Gao, Q. Meng, H. Sirringhaus and D. Zhu, Advanced Materials 25 (10), 1370 (2013).

11. H. Li, F. S. Kim, G. Ren and S. A. Jenekhe, Journal of the American Chemical Society $\mathbf{1 3 5}$ (40), 14920 (2013).

12. L.-K. Mao, J.-C. Hwang, T.-H. Chang, C.-Y. Hsieh, L.-S. Tsai, Y.-L. Chueh, S. S. Hsu, P.-C. Lyu and T.-J. Liu, Organic Electronics (2013). 
13. M. J. Robb, S. Y. Ku, F. G. Brunetti and C. J. Hawker, Journal of Polymer Science Part A: Polymer Chemistry 51 (6), 1263 (2013).

14. C. D. Easton and M. V. Jacob, Polymer Degradation and Stability 94 (4), 597 (2009).

15. C. D. Easton and M. V. Jacob, Thin Solid Films 517 (15), 4402 (2009).

16. C. D. Easton and M. V. Jacob, Journal of Applied Polymer Science 115 (1), 404 (2010).

17. C. D. Easton, M. V. Jacob, R. A. Shanks and B. F. Bowden, Chemical Vapor Deposition 15 (7-9), 179 (2009).

18. L. J. Anderson and M. V. Jacob, Applied Surface Science 256 (10), 3293 (2010).

19. K. Bazaka and M. V. Jacob, Materials Letters 63 (18-19), 1594 (2009).

20. M. V. Jacob, C. D. Easton, G. S. Woods and C. C. Berndt, Thin Solid Films 516 (12), 3884 (2008).

21. R. N. Wenzel, The Journal of Physical and Colloid Chemistry 53 (9), 1466 (1949).

22. S. Kurosawa, N. Kamo, N. Minoura and M. Muratsugu, Materials Science and Engineering: C 4 (4), 291 (1997).

23. S. Dalleau, E. Cateau, T. Bergès, J.-M. Berjeaud and C. Imbert, International journal of antimicrobial agents 31 (6), 572 (2008). 